\title{
Diffraction of sound by two finite-length cascade cylinders
}

\author{
Shinya Kijimoto, ${ }^{*}$ Hideo Suzuki, ${ }^{* *}$ and Shun Oguro** \\ *Department of Intelligent Machinery and Systems, Faculty of Engineering, \\ Kyushu University, 6-10-1, Hakozaki, Higashi-ku, Fukuoka, 812-8581 Japan \\ ** Ontek R\&D Co., Ltd., \\ 1-16-1, Hakusan, Midori-ku, Yokohama, 226-8507, Japan
}

(Received 6 June 1997)

\begin{abstract}
In order to investigate the influence of the sound intensity probe itself on the accuracy of measurement, a diffraction of the plane progressive wave by two finite-length cascade cylinders is calculated by the boundary element method (BEM). The sound pressure distribution around the cylinders is compared with the measured one. Both results agree well with each other. Frequency characteristics of the force that acts on the surface facing the gap between two cylinders are calculated for various probe model geometries. The force response shows deviations at high frequencies where the probe size becomes comparable to the wave length. This deviation mainly depends on the diameter of the probe.
\end{abstract}

Keywords: Diffraction, Sound intensity probe, Boundary element method

PACS number: 43. 20. Fn

\section{INTRODUCTION}

It was about 15 years ago when a measurement method of the acoustic intensity was made practicable. But there are still some technical problems that must be solved. It is one of such problems that how the microphone probe geometries affect measurement results. A presently used typical microphone probe has a pair of microphones facing to each other with a spacer between them. Because of this structure, sound must come through narrow crevices. When the probe is set in the plane progressive wave, a sound pressure level on the diaphragm is different from a sound pressure level of the plane progressive wave at high frequencies where the wavelength is on the same order to a size of the microphone probe. If the relation between the probe geometries and the sound pressure distribution on the diaphragm is known, a measurement error can be estimated theoretically.

In this paper, the microphone probe is modeled by two finite-length cascade cylinders. Frequency characteristics of a force that acts on the surface facing the gap are investigated for various model geometries. The reason why the force characteristics is investigated is that it may represent an electrical output response of the microphone. Even though an actual microphone probe has two gaps, only one gap is taken into account in this paper.

The present problem that must be solved is the sound analysis in the infinite space. The boundary element method ${ }^{1)}$ (BEM) is a suitable method for this purpose. To confirm a reliability of the calculation, a 1:5 scale model is made and the sound pressure distribution is measured near the surface of the model. Measurement results are compared with calculated ones.

\section{SOUND FIELD ANALYSIS BY BEM}

In this paper, the boundary element method (BEM) is used for the sound diffraction problem. This method is superior to the finite element method (FEM), that requires to divide not only the boundaries but also the space into many elements. 


\subsection{Fundamental Equations}

A sound pressure $P$ in an uniform acoustic medium is described by the Helmholtz's equation :

$$
\nabla^{2} P+k^{2} P=0
$$

where $k$ is a wavenumber.

The following equation can be written by the weighted residual technique.

$$
C_{i} P_{i}+\int_{\Gamma} P \frac{\partial f}{\partial n} d \Gamma=\int_{\Gamma} \frac{\partial P}{\partial n} f d \Gamma
$$

where $f$ is a fundamental solution, $n$ is a vector normal to the boundary $\Gamma$ and $C_{i}$ is a singular integral coefficient. For a three dimensional case the fundamental solution $f$ is given by

$$
f=\frac{1}{4 \pi r} \exp (-j k r) \text {. }
$$

\subsection{Discretization}

A boundary will be divided into $n$ linear elements. Equation (2) can be written for the $n$ elements ;

$$
C_{i} P_{i}+\sum_{j=1}^{n} \int_{\Gamma_{j}} P \frac{\partial f}{n} d \Gamma=\sum_{j=1}^{n} \int_{\Gamma_{j}} \frac{\partial P}{\partial n} f d \Gamma
$$

The integrals along elements in Eq. (4) are discretized by using the linear interpolation functions. So, Eq. (4) can be written as,

$$
\sum_{j=1}^{n} H_{i j} P_{j}=\sum_{j=1}^{n} G_{i j} \frac{\partial P_{j}}{\partial n}
$$

Equation (5) can be expressed in matrix form as ;

$$
[H]\{P\}=[G]\left\{\frac{\partial P}{\partial n}\right\}
$$

by solving Eq. (6) the values of $\{P\}$ and $\{\partial P / \partial n\}$ on the boundary are known.

\subsection{Diffraction Problem}

A plane propagating wave impinges on the two rigid cylinders with an oblique angle. A common method used to solve diffraction problems is as follows :

(1) Calculate the velocity component of the incident wave normal to the cylinder surface assuming that the cylinders do not exist.

(2) Solve the radiation problem assuming that the cylinders vibrate with the velocity distribution.

(3) Superimpose the pressure distributions of the incident wave and the one obtained in (2) so that the velocity distribution normal to the cylinder surface is zero.

\section{MICROPHONE PROBE}

Figure 1 shows a simplified model of the micro- phone probe. The probe consists of two cylinders (I and II) that have the same center axis and the same diameter. In Fig.1, $S_{1}$ is the sensing area corresponding to a microphone diaphragm and point $\mathrm{O}$ is the center of surface $\mathrm{S}_{2}$, a base surface of

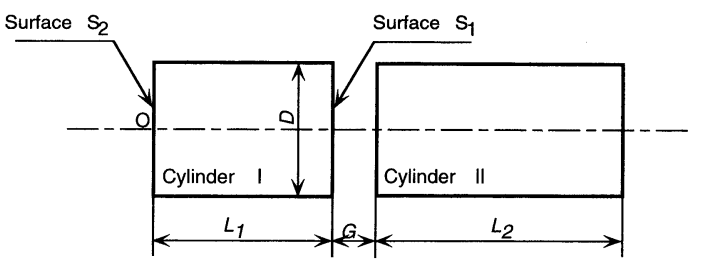

Fig. 1 Microphone probe model.

Table 1 Four microphone probe models for BEM analysis.

\begin{tabular}{ccccc}
\hline & $D$ & $L_{1}$ & $L_{2}$ & $G$ \\
\hline probe-A & 15 & 20 & 30 & 1.5 \\
\hline probe-B & 10 & 20 & 30 & 1.5 \\
\hline probe-C & 15 & 10 & 30 & 1.5 \\
\hline probe-D & 15 & 20 & 30 & 1.0 \\
\hline & & & \multicolumn{3}{c}{ unit : $\mathrm{mm}$}
\end{tabular}

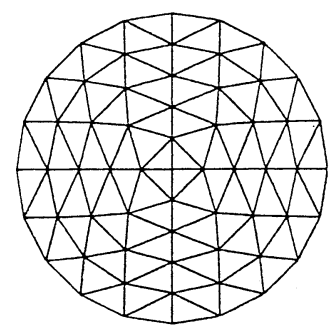

(a) base

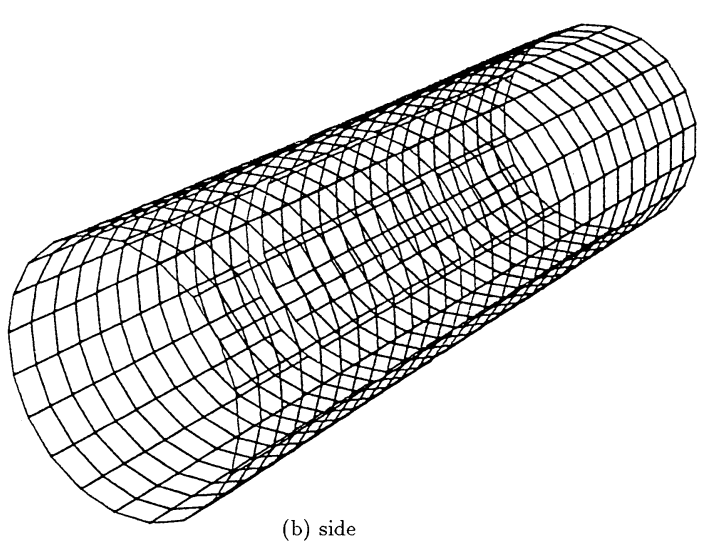

Fig. 2 BEM division. 


\section{S. KIJIMOTO et al. : DIFFRACTION OF SOUND BY TWO CASCADE CYLINDERS}

cylinder I. $D$ is a diameter of two cylinders, $L_{1}$ is a length of cylinder I, $L_{2}$ is a length of cylinder II and $G$ is a gap width. Calculations were carried out for four models. Each variables of these models are shown in Table 1. BEM division is shown in Fig. 2.

\section{SOUND PRESSURE DISTRIBUTION AROUND CYLINDERS}

It is important to know the sound pressure distri-

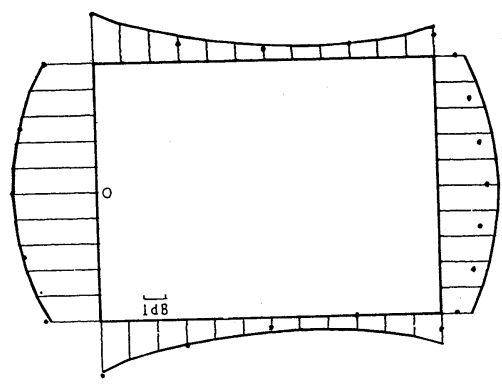

(a) Incident angle $0^{\circ}$, Frequency $1 \mathrm{kHz}$

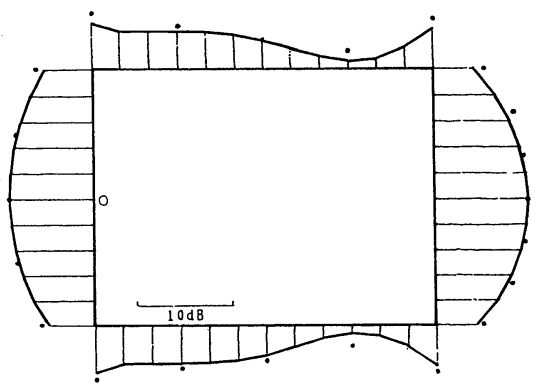

(b) Incident angle $0^{\circ}$, Frequency $2 \mathrm{kHz}$

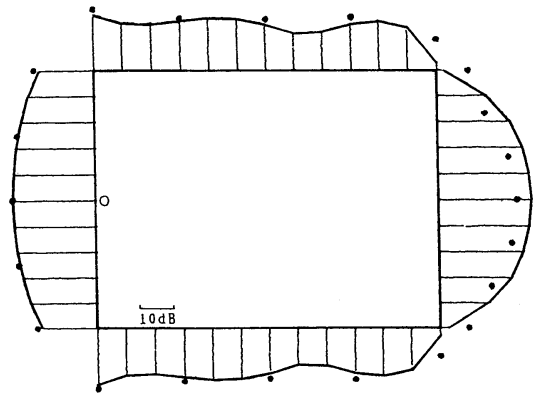

(c) Incident angle $0^{\circ}$, Frequency $4 \mathrm{kHz}$ bution around the microphone since a sensitivity of the microphone is influenced by the sound pressure distribution on the diaphragm. In order to evaluate a precision of the calculation, a magnification model is made and measurement values are compared with calculated ones.

\subsection{Calculated Results}

Figure 2 shows the BEM model using triangle and quadrilateral linear elements. The BEM model has

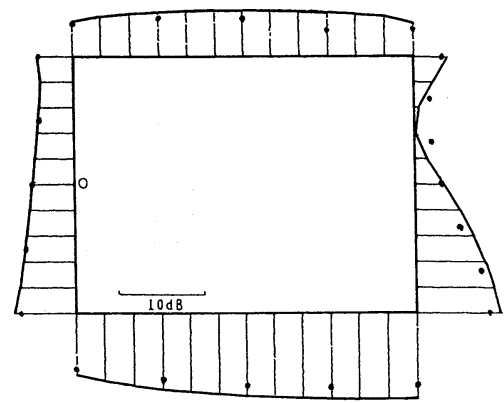

(d) Incident angle $90^{\circ}$, Frequency $1 \mathrm{kHz}$

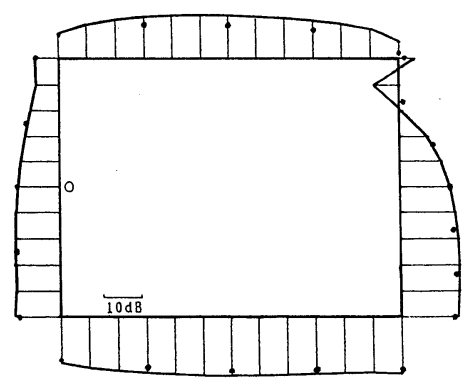

(e) Incident angle $90^{\circ}$, Frequency $2 \mathrm{kHz}$

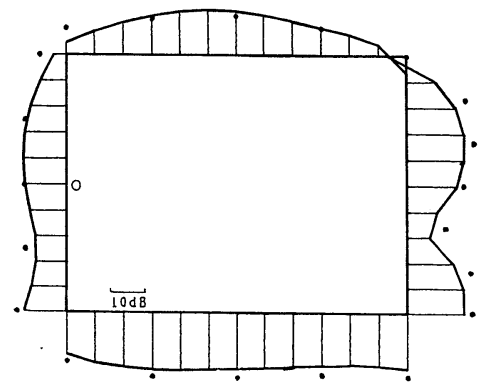

(f) Incident angle $90^{\circ}$, Frequency $4 \mathrm{kHz}$

Fig. 3 Calculated (solid line) and measured (dot) sound pressure distributions. 
744 nodes, 940 elements. At the highest frequency of the analysis, the wave length is four times of the maximum length of elements. Calculated results normalized to the sound pressure level at point $\mathrm{O}$ are shown by solid lines in Fig. 3(a-f). A $1 \mathrm{~dB}$ or 10 $\mathrm{dB}$ scale is shown by a straight line on each figures (a-f) in Fig. 3.

When the incident angle is $0^{\circ}$, the sound pressure level at the center of surface $S_{1}$ is about $10 \mathrm{~dB}$ higher than the one at the edge of the same surface at $4 \mathrm{kHz}$. Similar results are shown on the surface $\mathrm{S}_{2}$, but the sound pressure difference between at the center and at the edge is less than half of the difference on the surface $S_{1}$. A distribution on the side of cylinder I shows rather complex patterns. In the case of the $90^{\circ}$ incident angle, it is very interesting that the distribution is not symmetric. If the diaphragm has a directional tension or if it is not parallel with the sensing electrode behind it, the sensitivity and phase characteristics of the microphone may change by the angle of the incident wave around the cylinder axis.

\subsection{Experimental Results}

The magnification model is five times larger in size than the standard calculation model (probe-A). Measurement frequencies are 1,2 and $4 \mathrm{kHz}$, which correspond to 5,10 and $20 \mathrm{kHz}$ with probe-A, respectively. In the experiment, two incident angles $\left(0^{\circ}\right.$

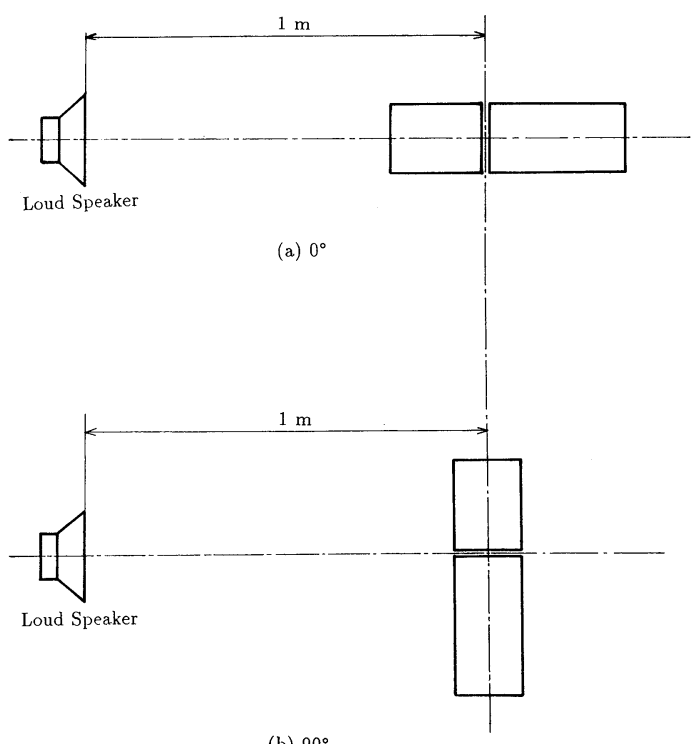

(b) $90^{\circ}$

Fig. 4 Measurement setup of the sound pressure distribution around the probe model. and $90^{\circ}$ ) are selected as shown in Fig. 4. The experiment was carried out in an anechoic room. The experimental results are indicated by dots in Fig. 3. Each dots show decibel values normalized to the value at point $\mathrm{O}$. As seen in Fig. 3, calculated results agree very well with experimental ones. But on the surface $S_{1}$, the difference is slightly larger than the one of the other parts. One possible reason of this difference is that a microphone of $5 \mathrm{~mm}$ in diameter is inserted into the $7.5 \mathrm{~mm}$ gap for measurement.

\section{FREQUENCY CHARACTERISTICS OF THE FORCE ACTING ON SURFACE $S_{1}$}

A frequency response of the force acting on surface $S_{1}$ is calculated by BEM for the cases shown in Table 1. The boundary element division is the same as the one used in Section 4. The frequency response is calculated at 16 frequencies from 1,000 $\mathrm{Hz}$ through $10,000 \mathrm{~Hz}$ and the incident angles are $0^{\circ}$, $30^{\circ}, 60^{\circ}$ and $90^{\circ}$. Results about four models are shown in Fig. 5(a-d).

Results of probe-A (Fig. 5(a)) shows large variations at high frequencies. If the incident angle is $0^{\circ}$, the force response rises about $2.5 \mathrm{~dB}$ at $7 \mathrm{kHz}$. On the other hand, if the incident angle is $60^{\circ}$, it declines about $1.6 \mathrm{~dB}$ at the same frequency. Simply assuming that the force response on the diaphragm corresponds to the sound pressure characteristic of the microphone, these results show that the measurement accuracy of the sound intensity is significantly dependent on the incident angle.

Figure 5(b) shows the results of probe-B. A diameter of this model is $10 \mathrm{~mm}$. At $7 \mathrm{kHz}$, the force response for zero incident angle rises $1.2 \mathrm{~dB}$, and it declines $1.2 \mathrm{~dB}$ when the incident angle is $60^{\circ}$. A comparison of Figs. 5(a) and 5(b) shows that the variation of the force response is highly dependent on the cylinder diameter.

Figure 5(c) shows the results of probe-C. A difference between probe- $\mathrm{C}$ and probe- $\mathrm{A}$ is the length of cylinder I $\left(L_{1}\right)$. The force response of probe- $\mathrm{C}$ is not so different from the one of probe-A. These results show that the length of the front cylinder I has a small influence on the frequency response.

Figure 5(d) shows the results of probe-D. The force response shows less variation when the gap width $G$ is changed to $1 \mathrm{~mm}$. 


\section{S. KIJIMOTO et al. : DIFFRACTION OF SOUND BY TWO CASCADE CYLINDERS}

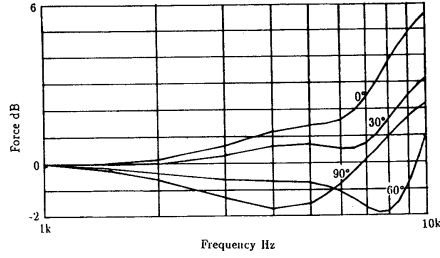

(a) probe-A

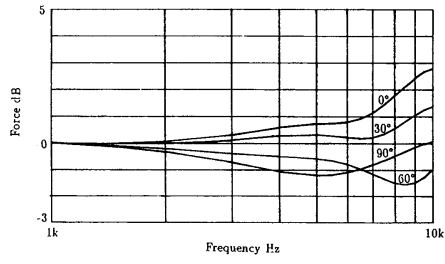

(b) probe-B

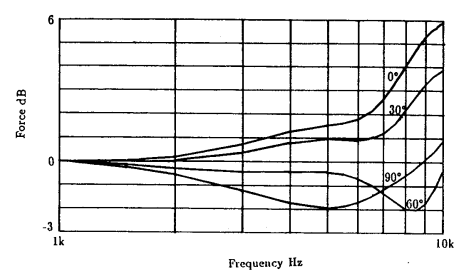

(c) probe-C

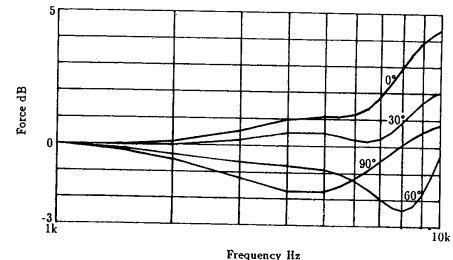

(d) probe-D

Fig. 5 Frequency responses of the force acting on suface $S_{1}$.

The above results show that the diameter of the probe has a great influence on the force response. The diameter of the probe is a very important parameter to keep a measurement accuracy within a required error limit.

\section{CONCLUSIONS}

In this paper, a diffraction of a plane incident wave around two cascade cylinders was investigated by BEM. First, a sound pressure distribution around the probe was calculated and compared with measurement values using a magnification model. The accuracy of the calculation was indirectly confirmed by the agreement with the measurement results. The force distribution on the surface facing the gap is not uniform, indicating that it must be taken into account when a microphone sensitivity is theoretically estimated. This nonuniformity also raises a question that the microphone sensitivity may be dependent on the angle around the axis even if the incident angles are the same.

Secondly, the force response on the diaphragm was investigated. It was confirmed that the force varies with a frequency. This means that even if the sensitivity of the microphone is uniform, the output of the probe shows the frequency dependence. When the diameter of the probe is $15 \mathrm{~mm}$, the force response for the case of zero incident angle rises by $2 \mathrm{~dB}$ at $7 \mathrm{kHz}$. But on the other hand, if the incident angle is $60^{\circ}$ it declines nearly $2 \mathrm{~dB}$ at the same frequency. If the diameter of the probe is reduced, a variation of the force response decreases considerably. This means that the diameter has a great influence on the force response compared with a cylinder length or a gap width.

In this paper, only one gap was taken into account. An actual sound intensity probe has two gaps. Phase differences of the force acting on the two gaps is an essential factor for the intensity measurement. This will be investigated in the second step of this research.

\section{REFERENCES}

1) C. A. Brebbia, The Boundary Element Method for Engineers (Pentech Press, London, Plymouth, 1978). 